\title{
Vaccinations Administered During Off-Clinic Hours at a National Community Pharmacy: Implications for Increasing Patient Access and Convenience
}

\author{
Jeffery A. Goad, PharmD, MPH \\ Michael S. Taitel, $P b D^{2}$ \\ Leonard E. Fensterbeim, MPH \\ Adam E. Cannon, $\mathrm{MPH}^{2}$ \\ 'University of Southern California, School \\ of Pharmacy, Los Angeles, California \\ ${ }^{2}$ Walgreen Company, Deerfield, Illinois
}

\begin{abstract}
PURPOSE Approximately 50,000 adults die annually from vaccine-preventable diseases in the United States. Most traditional vaccine providers (eg, physician offices) administer vaccinations during standard clinic hours, but community pharmacies offer expanded hours that allow patients to be vaccinated at convenient times. We analyzed the types of vaccines administered and patient populations vaccinated during off-clinic hours in a national community pharmacy, and their implications for vaccination access and convenience.
\end{abstract}

METHODS We retrospectively reviewed data for all vaccinations given at the Walgreens pharmacy chain between August 2011 and July 2012. The time of vaccination was categorized as occurring during traditional hours (9:00 AM-6:00 PM weekdays) or off-clinic hours, consisting of weekday evenings, weekends, and federal holidays. We compared demographic characteristics and types of vaccine. We used a logistic regression model to identify predictors of being vaccinated during off-clinic hours.

RESULTS During the study period, pharmacists administered 6,250,402 vaccinations, of which $30.5 \%$ were provided during off-clinic hours: $17.4 \%$ were provided on weekends, $10.2 \%$ on evenings, and $2.9 \%$ on holidays. Patients had significantly higher odds of off-clinic vaccination if they were younger than 65 years of age, were male, resided in an urban area, and did not have any chronic conditions.

CONCLUSIONS A large proportion of adults being vaccinated receive their vaccines during evening, weekend, and holiday hours at the pharmacy, when traditional vaccine providers are likely unavailable. Younger, working-aged, healthy adults, in particular, accessed a variety of immunizations during off-clinic hours. With the low rates of adult and adolescent vaccination in the United States, community pharmacies are creating new opportunities for vaccination that expand access and convenience.

Ann Fam Med 2013;429-436. doi:10.1370/afm.1542.

\section{INTRODUCTION}

The National Foundation for Infectious Diseases estimates that 50,000 adults die annually from vaccine-preventable diseases in the United

States. ${ }^{1}$ Although childhood vaccination rates exceed $90 \%$ for most
ecommended vaccines ${ }^{2}$ adult vaccination rates are all below national goals.

Conflicts of interest: Dr Taitel, Mr Fensterbeim, and $\mathrm{Mr}$ Cannon are employees of Walgreen Co. Dr Goad serves on the speaker's bureau for Merck and Co, Inc.

\section{CORRESPONDING AUTHOR}

Leonard E. Fensterheim, MPH

Walgreen Co

1415 Lake Cook Rd, MS L444

Deerfield, IL 60015

leonard.fensterheim@walgreens.com Adult immunization rates for recommended vaccines range from a low of $14 \%$ for the herpes zoster vaccine to a high of $64 \%$ for the tetanus vaccine (the rate for the tetanus, diphtheria, and pertussis vaccine is $8.2 \%$ ). ${ }^{3}$ Influenza vaccination is recommended for all individuals aged 6 months and older. In a given season, approximately 23,000 influenza-associated deaths occur, mostly in adults. ${ }^{4}$ Adult influenza vaccination rates vary widely, but they average just $40.5 \%$, with the highest rates seen in those aged 65 years and older $(69.6 \%)^{5}$

Community pharmacies are uniquely positioned to increase immunization rates in the United States. In a 2009 survey, only $20 \%$ to $30 \%$ of 
internists and family physicians stocked all vaccines recommended by the Centers for Disease Control and Prevention (CDC), with nearly $80 \%$ not planning to increase their offerings and $2 \%$ planning to stop carrying them altogether. ${ }^{6}$ The CDC refers to pharmacies as nontraditional locations for receiving vaccines, offering advantages such as community-based locations, access, and convenience. ${ }^{7}$ In the 2010-2011 influenza season, $18.4 \%$ of people were vaccinated in a store $(\mathrm{eg}$, supermarket or drug store). ${ }^{8}$ Evidence in the medical literature suggests that pharmacies have the capacity to influence previously difficult-to-reach populations, including the medically underserved. ${ }^{9-13}$ Pharmacies may be especially effective in immunizing high-risk older adults, who are more likely to use pharmacy services for prescription medication than the general population. ${ }^{14}$ Little is known, however, about the vaccination behaviors of younger working-aged adults.

Whereas most traditional providers (eg, primary care physician offices) administer vaccinations during traditional clinic hours (9:00 AM to 5:00 PM, Monday through Friday) ${ }_{1}^{15}$ community pharmacies often offer expanded hours that allow patients to receive vaccinations at more convenient times. The objectives of this study were to analyze the types of vaccines administered and patient populations vaccinated during off-clinic hours in a national community pharmacy, and implications for vaccination access and convenience.

\section{METHODS}

\section{Study Design and Population}

We retrospectively analyzed all vaccinations administered at the Walgreens pharmacy chain between August 1, 2011, and July 31, 2012. Pharmacy records for all immunizations administered at the pharmacy during the study period were identified. At the time of the study, there were more than 7,800 Walgreens pharmacies located in 49 states (North Dakota did not have any), the District of Columbia, and Puerto Rico that provided immunization services. More than 26,000 Walgreens pharmacists have completed the American Pharmacist Association's Pharmacy-Based Immunization Delivery Training.

We categorized the time of vaccination as occurring during traditional hours (9:00 AM to 6:00 PM weekdays) or off-clinic hours, consisting of weekdays 6:00 PM to 9:00 AM, weekends, and 10 federal holidays-New Year's Day, birthday of Martin Luther King, Jr, Washington's Birthday, Memorial Day, Independence Day, Labor Day, Columbus Day, Veterans Day, Thanksgiving Day, and Christmas Day. ${ }^{16} \mathrm{We}$ identified the type of vaccine from the Food and Drug Administration's 11-digit National Drug Code.
Demographic characteristics of the patients vaccinated during the study period were noted, including their age and sex, urban vs other residence, and any chronic conditions, based on the Medical Condition Code as developed by Medi-Span. ${ }^{17}$ Population density area of residence was classified as metropolitan, micropolitan, small town, or rural based on zip codes of the patient address according to the Rural Urban Commuting Area codes. ${ }^{18}$

We classified patients as insured (having private or government insurance, or redeeming a voucher to receive a vaccination) or uninsured at time of vaccination. Patients were classified as receiving a vaccination in a state in which pharmacists are authorized to administer either (1) all CDC-recommended immunizations per protocol or with a patient-specific prescription, or (2) selected CDC-recommended immunizations limited to influenza, pneumonia, zoster, or some combination thereof. ${ }^{19}$ Vaccine type was classified as either a vaccine included on the CDC adult or adolescent recommended immunization table ${ }^{20,21}$ or other vaccine approved by the Food and Drug Administration (eg, for travel), consisting of typhoid, yellow fever, rabies, and Japanese encephalitis. We also noted the number of vaccines a patient received during the year as well as whether patients picked up a prescription medication at the time of vaccination.

\section{Statistical Analysis}

We first used univariate analyses to describe the temporal distribution of vaccinations, type of vaccine, and patient characteristics in the study population, and their use of traditional and off-clinic hours for vaccination. Percent of traditional clinic and off-clinic use were detailed for each subgroup of patients identified by the characteristics studied.

We conducted logistic regression analysis using the patient characteristics and pharmacy use variables. The model consisted of age category, sex, urban classification, presence of 1 or more chronic conditions, patient insurance status, state-level pharmacist immunization privilege for CDC-recommended vaccinations, vaccine type, number of vaccinations received, and whether the patient picked up a prescription medication at the time of vaccination. These factors were hypothesized to be predictors of pharmacy-based immunization services. The results of the model are presented as odds ratios (ORs) predicting the likelihood a patient with a given characteristic would use off-clinic hours for their vaccination.

Statistical significance was assessed at the $\alpha=.05$ level. All data analysis was performed with SAS 9.2 software (SAS Institute Inc). This study was approved by the Quorum institutional review board under protocol 26510 . 


\section{RESULTS}

\section{Distribution of Vaccinations}

During the study period, 6,250,402 vaccinations were administered to the study population. Of these, $85.0 \%$ were for influenza and $15.0 \%$ were for other vaccinepreventable diseases: herpes zoster (shingles); pneumonia; tetanus, diphtheria, and acellular pertussis (Tdap); meningitis; typhoid; hepatitis A and/or B (hepatitis); human papillomavirus (HPV); measles, mumps, and rubella $(\mathrm{MMR})$; varicella zoster (chicken pox); yellow fever; rabies; polio; and Japanese encephalitis - in order of greatest to least frequency.

By time, 4,344,050 (69.5\%) of the immunizations were administered during traditional clinic hours, and $1,906,352(30.5 \%)$ were administered during off-clinic hours (Table 1 ). Of the total vaccinations administered, $17.4 \%$ were on weekends, $10.2 \%$ on evenings, and $2.9 \%$ on holidays. There were $1,093,128$ vaccinations (17.5\% of all vaccinations) administered during lunch hours, defined as weekdays 11:00 AM to 1:00 PM, and 150,366 vaccinations (2.4\% of all vaccinations; $7.9 \%$ of off-clinic vaccinations) were administered during overnight hours (10:00 PM to 9:00 AM) (Figure 1). The smallest number of vaccinations, $1,680(0.03 \%$ of all vaccinations), was administered between 3:00 AM and 4:00 AM.
Table 1. Temporal Distribution of All Vaccinations Administered

\begin{tabular}{|c|c|c|}
\hline Time of Vaccination & Number & Percent \\
\hline Traditional clinic hours $^{a}$ & $4,344,050$ & 69.50 \\
\hline Off-clinic hours & $1,906,352$ & 30.50 \\
\hline Evenings $^{b}$ & 636,973 & 10.19 \\
\hline Weekends & $1,087,028$ & 17.39 \\
\hline Federal holidays ${ }^{c}$ & 182,351 & 2.92 \\
\hline Labor Day & 18,872 & 0.30 \\
\hline Columbus Day & 91,008 & 1.46 \\
\hline Veterans Day & 42,607 & 0.68 \\
\hline Thanksgiving Day & 1,436 & 0.02 \\
\hline Christmas Day & 6,808 & 0.11 \\
\hline New Year's Day & 9,085 & 0.15 \\
\hline Birthday of Martin Luther King, Jr & 6,592 & 0.11 \\
\hline Washington's Birthday & 3,614 & 0.06 \\
\hline Memorial Day & 1,165 & 0.02 \\
\hline Independence Day & 1,164 & 0.02 \\
\hline Total & $6,250,402$ & 100.00 \\
\hline \multicolumn{3}{|c|}{$\begin{array}{l}\text { Notes: Vaccinations offered included influenza; herpes zoster (shingles); pneumo- } \\
\text { nia; tetanus, diphtheria, and acellular pertussis (Tdap); typhoid; hepatitis A and/or } \\
\text { B; human papillomavirus (HPV); meningitis; measles, mumps, and rubella (MMR); } \\
\text { varicella zoster (chicken pox); yellow fever; rabies; polio; and Japanese encephalitis }\end{array}$} \\
\hline $\begin{array}{l}\text { a Weekdays 9:00 AM to 6:00 PM. } \\
\text { b Weekdays 6:00 PM to 9:00 AM. } \\
\text { ' Holidays that occurred during a weekend }\end{array}$ & vere classified a & holidays. \\
\hline \multicolumn{3}{|c|}{ Source: Walgreens pharmacy claims data, August 2011 through July 2012.} \\
\hline
\end{tabular}

\section{Figure 1. Hourly distributions of vaccinations.}

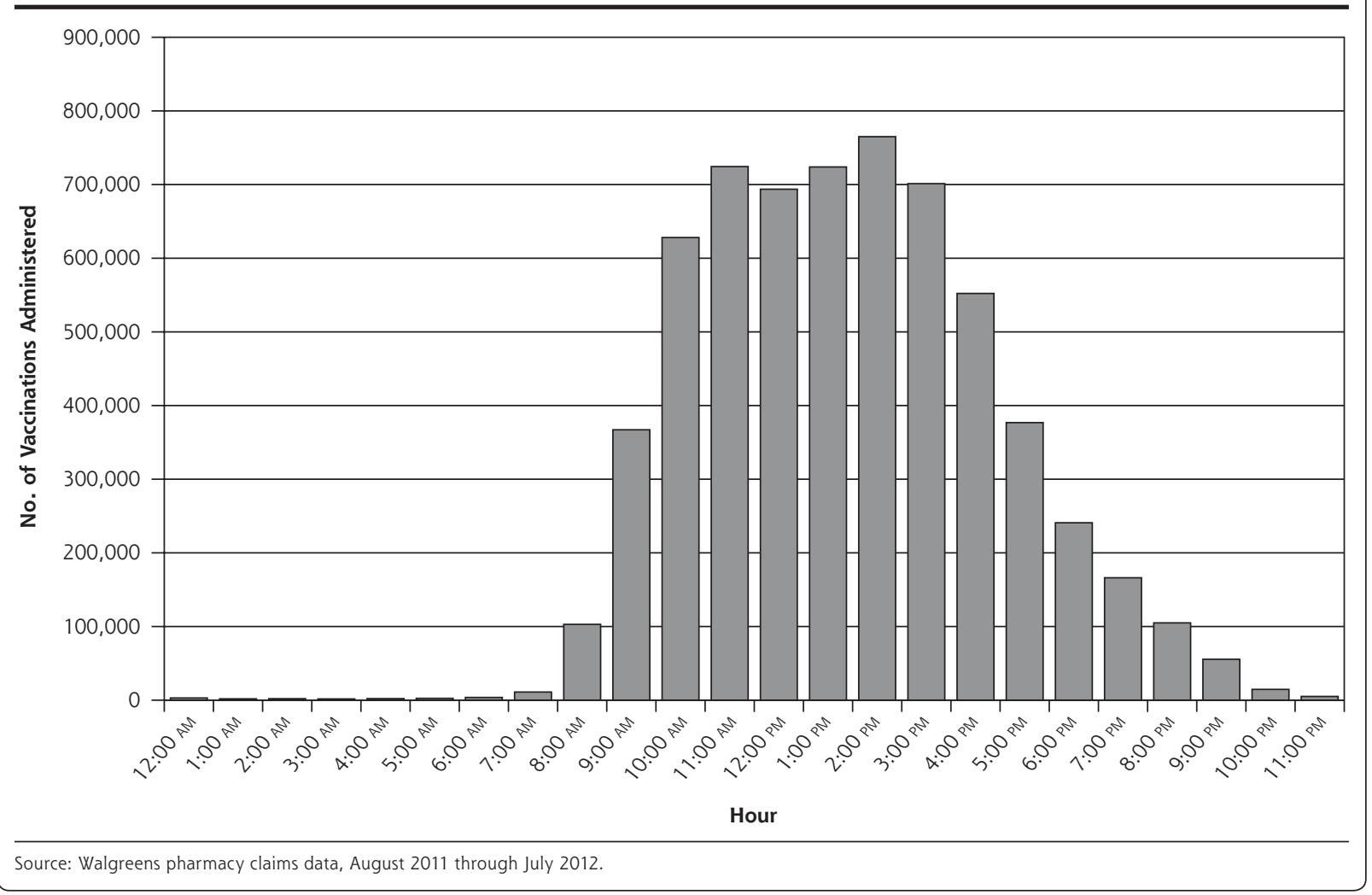


The vaccine types with the greatest proportion administered during off-clinic hours were typhoid (41.5\%), Tdap (34.5\%), HPV (33.8\%), varicella zoster $(31.6 \%)$, influenza $(31.5 \%)$, hepatitis $(31.4 \%)$, and yellow fever (30.8\%) (Figure 2).

\section{Demographics of Study Population}

Table 2 displays traditional clinic and off-clinic hour vaccinations administered by patient characteristics and pharmacy use. For the $5,787,970$ unique patients vaccinated, the average age was 60.8 years during traditional hours and 53.1 years during off-clinic hours. Among patients aged younger than 65 years, 39.0\% received their vaccinations during off-clinic hours. About onethird of both men (32.5\%) and women (30.4\%) did so.

Among patients stratified by the population density classifications, the largest proportion receiving vaccinations during off-clinic hours resided in metropolitan areas (32.3\%). The proportion vaccinated during offclinic hours was similar for patients without vs with chronic conditions (31.9\% vs $30.5 \%$ ) but greater for uninsured patients vs insured patients (37.6\% vs $29.7 \%)$.

\section{Pharmacy Use of Study Population}

Among patients who received a travel or other vaccine not routinely recommended by the CDC, $40.3 \%$ were vaccinated during off-clinic hours, compared with $31.3 \%$ of patients receiving a CDC-recommended vaccination. The proportion of patients vaccinated during off-clinic hours was generally similar among patients receiving multiple vs single vaccines during the year (33.9\% vs $31.1 \%)$ and among patients also picking up a prescription as compared with those just receiving a vaccine $(31.5 \%$ vs $31.3 \%)$.

\section{Predictors of Vaccination During Off-Clinic Hours}

Table 3 displays the multivariate adjusted ORs with 95\% Cls for vaccination during the off-clinic hours. With respect to patient characteristics, patients were more likely to be vaccinated during off-clinic hours if they were male vs female $(\mathrm{OR}=1.080 ; 95 \% \mathrm{CI}$, 1.076-1.084) and if they resided in a metropolitan area $(\mathrm{OR}=1.336 ; 95 \% \mathrm{CI}, 1.319-1.353)$. On the other hand, patients were less likely to be vaccinated during off-clinic hours if they were aged 65 years or older vs younger $(\mathrm{OR}=0.447 ; 95 \% \mathrm{CI}, 0.445-0.449)$, had at least 1 chronic condition $(\mathrm{OR}=0.980 ; 95 \% \mathrm{CI}, 0.976-0.983)$, or had insurance $(\mathrm{OR}=0.991 ; 95 \% \mathrm{CI}, 0.987-0.996)$.

With respect to pharmacy characteristics and vaccination-related factors, patients were more likely to use off-clinic hours if they were vaccinated in states that authorize pharmacists to administer all CDC-recommended vaccines vs states that authorize pharmacists to administer only influenza, pneumonia, and zoster vaccines $(\mathrm{OR}=1.044 ; 95 \% \mathrm{CI}, 1.040-1.049)$, if they received travel vaccines vs routine CDC-recommended vaccines $(\mathrm{OR}=1.166 ; 95 \% \mathrm{CI}, 1.137-1.195)$, and if they received multiple vaccines at the pharmacy during the year $(\mathrm{OR}=1.353 ; 95 \% \mathrm{CI}, 1.343-1.363)$. Picking up a prescription medication at the time of vaccination did not significantly influence off-clinic use $(\mathrm{OR}=1.000$; 95\% CI, 0.994-1.005).

\section{DISCUSSION}

\section{Key Findings}

In our study of more than 6 million vaccinations administered in more than 7,500 pharmacies nationwide, we focused on immunization services during hours outside of traditional medical clinic hours. The off-clinic population received immunizations from 6:00 PM to 9:00 AM, Monday through Friday, and any time on weekends and US federal holidays. Results show that approximately $30 \%$ of the study population received 1 or more vaccinations during these off-clinic hours. Almost 200,000 patients were vaccinated on a federal holiday, most often Columbus Day and Veterans Day. As these holidays occur in October and

\section{Figure 2. Vaccinations with the greatest proportion administered during off-clinic hours.}

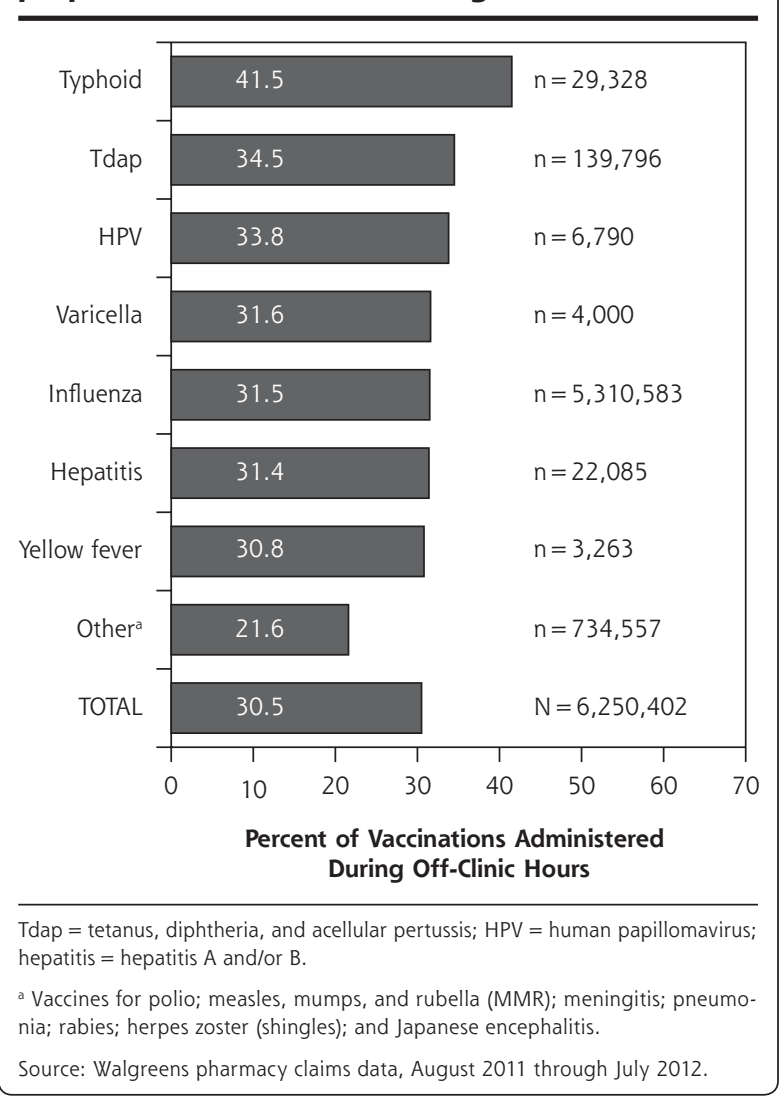


November, respectively, and coincide with typical influenza season in the northern hemisphere, expansion of pharmacy hours to include these holidays facilitates access and convenience for influenza vaccinations (as well as others) during a time when traditional providers are not likely available.

Table 2. Vaccinations Administered According to Time, by Patient Characteristics and Pharmacy Use

\begin{tabular}{|c|c|c|c|}
\hline \multirow[b]{2}{*}{ Measure } & \multicolumn{2}{|c|}{ Time of Vaccination } & \multirow[b]{2}{*}{$\begin{array}{c}\text { Total Cohort } \\
\text { No. }(\%) \\
(\mathrm{N}=5,787,970)\end{array}$} \\
\hline & $\begin{array}{c}\text { Traditional } \\
\text { Clinic Hours, \% } \\
(n=3,976,029)\end{array}$ & $\begin{array}{c}\text { Off-Clinic } \\
\text { Hours, } \% \\
\text { (n }=1,811,941)\end{array}$ & \\
\hline \multicolumn{4}{|l|}{ Age category ${ }^{a}$} \\
\hline$<18$ years & 48.9 & $51.1^{\mathrm{b}}$ & $191,594(3.3)$ \\
\hline $18-49$ years & 59.0 & $41.0^{\mathrm{b}}$ & $1,504,681(26.0)$ \\
\hline $50-64$ years & 64.8 & $35.2^{\mathrm{b}}$ & $1,452,577(25.1)$ \\
\hline$\geq 65$ years & 77.8 & 22.2 & $2,639,118(45.6)$ \\
\hline \multicolumn{4}{|l|}{ Sex } \\
\hline Male & 67.5 & $32.5^{\mathrm{b}}$ & $2,468,472(42.7)$ \\
\hline Female & 69.6 & 30.4 & $3,319,498(57.4)$ \\
\hline \multicolumn{4}{|l|}{$\begin{array}{l}\text { Population density of patient } \\
\text { address }\end{array}$} \\
\hline Metropolitan & 67.8 & $32.3^{b}$ & $4,863,492(84.0)$ \\
\hline Micropolitan & 73.2 & 26.8 & $583,455(10.1)$ \\
\hline Small town & 74.2 & 25.8 & $207,270(3.6)$ \\
\hline Rural & 74.7 & 25.4 & $133,753(2.3)$ \\
\hline \multicolumn{4}{|l|}{ Chronic conditions $^{d}$} \\
\hline 0 & 68.1 & $31.9^{b}$ & $3,435,597(59.4)$ \\
\hline$\geq 1$ & 69.5 & 30.5 & $2,352,373(40.6)$ \\
\hline \multicolumn{4}{|l|}{ Insurance status } \\
\hline Insured & 70.3 & 29.7 & $4,605,207(79.6)$ \\
\hline Uninsured & 62.4 & $37.6^{\mathrm{b}}$ & $1,182,763(20.4)$ \\
\hline \multicolumn{4}{|l|}{ State-level pharmacist privilege ${ }^{e}$} \\
\hline All CDC recommended & 68.0 & $32.0^{\mathrm{b}}$ & $3,973,699(68.7)$ \\
\hline Selected CDC recommended & 70.3 & 29.7 & $1,814,271(31.4)$ \\
\hline \multicolumn{4}{|l|}{ Vaccine type ${ }^{f}$} \\
\hline CDC recommended & 68.7 & 31.3 & $5,756,905$ (99.5) \\
\hline Travel/other & 59.7 & $40.3^{b}$ & $31,065(0.5)$ \\
\hline \multicolumn{4}{|l|}{ Number of vaccines during year } \\
\hline 1 & 68.9 & 31.1 & $5,435,657(93.9)$ \\
\hline$\geq 2$ & 66.1 & $33.9^{b}$ & $352,313(6.1)$ \\
\hline \multicolumn{4}{|l|}{ Pharmacy use } \\
\hline Vaccination only & 68.7 & 31.3 & $5,031,745(86.9)$ \\
\hline Vaccination and prescription(s) & 68.5 & $31.5^{\mathrm{b}}$ & $756,225(13.1)$ \\
\hline Total & 68.7 & 31.3 & $5,787,970(100.0)$ \\
\hline
\end{tabular}

CDC $=$ Centers for Disease Control and Prevention.

a Calculated from patient's age on date of vaccination. Mean age was 60.8 years in the traditional clinic hours group and 53.1 years in the off-clinic hours group.

b Percentage is greater than the total off-clinic hours average of $31.3 \%$.

' Determined from patient zip code and Rural Urban Commuting Area (RUCA) codes. ${ }^{18}$

d Determined from Medical Condition Codes ascertained from patient prescriptions for chronic medications.

e Authorized per protocol or with patient-specific prescription for CDC-recommended vaccines.

f CDC-recommended vaccinations: influenza; herpes zoster (shingles); pneumonia; tetanus, diphtheria, and acellular pertussis (Tdap); hepatitis A and/or B (hepatitis); human papillomavirus (HPV); meningitis; measles, mumps, and rubella (MMR); varicella zoster (chicken pox); and polio. Travel/other vaccines: typhoid, yellow fever, rabies, and Japanese encephalitis.

Source: Walgreens pharmacy claims data, August 2011 through July 2012.
Results of this study also illustrate the access and convenience that community pharmacies provide for the typical working-aged population (aged 18-49 years). More than 1 million vaccinations took place during lunch hours (11:00 AM to 1:00 PM), possibly demonstrating use of these hours by patients working traditional workday hours of 9:00 AM to 5:00 PM. For this population, community pharmacies may offer a more convenient and time-efficient avenue for vaccination than physicians' offices. Furthermore, the more than 150,000 vaccinations that occurred during the overnight hours of 10:00 PM to 9:00 AM demonstrate the value that 24 -hour community pharmacies may provide to nightshift workers. According to the US Bureau of Labor Statistics, 15 million Americans work during overnight hours, either on a regular or occasional basis. ${ }^{22}$ For this population, 24-hour pharmacies may provide immediate access to vaccinations during their break time. In the workplace, absenteeism and productivity have been shown to improve with influenza vaccination. ${ }^{23,24}$ Allowing the working population to use convenient times outside of respective work hours to be vaccinated may thus increase vaccination rates and improve productivity.

Individuals receiving travel vaccines used off-clinic hours approximately $40 \%$ of the time. Travelers who plan to visit developing countries should take a number of preventive measures, including getting relevant vaccines. Some vaccines, such as the yellow fever vaccine, are mandated for country entry as determined by the World Health Organization. Other vaccine-preventable diseases, such as typhoid fever, have known country-specific risks, such as travel to the Indian subcontinent, but are often not given, resulting in numerous preventable cases of the disease. ${ }^{25} \mathrm{It}$ is estimated that only about $14 \%$ and $11 \%$ of travelers receive the hepatitis $\mathrm{A}$ and tetanus vaccines, 


\section{Table 3. Adjusted Associations of Patient Characteristics and Pharmacy Use With Vaccination During Off-Clinic Hours}

\begin{tabular}{|c|c|c|}
\hline Characteristic & Odds Ratio & $95 \% \mathrm{Cl}$ \\
\hline \multicolumn{3}{|l|}{ Age category ${ }^{b}$} \\
\hline$<65$ years & Referent & - \\
\hline$\geq 65$ years & $0.447^{c}$ & $0.445-0.449$ \\
\hline \multicolumn{3}{|l|}{ Sex } \\
\hline Female & Referent & - \\
\hline Male & $1.080^{c}$ & $1.076-1.084$ \\
\hline \multicolumn{3}{|l|}{$\begin{array}{l}\text { Population density of patient } \\
\text { address }^{d}\end{array}$} \\
\hline Metropolitan & $1.336^{c}$ & $1.319-1.353$ \\
\hline Micropolitan & $1.080^{c}$ & $1.065-1.095$ \\
\hline Small town & 1.008 & $0.992-1.024$ \\
\hline Rural & Referent & - \\
\hline \multicolumn{3}{|l|}{ Chronic conditions ${ }^{\mathrm{e}}$} \\
\hline 0 & Referent & - \\
\hline$\geq 1$ & $0.980^{c}$ & $0.976-0.983$ \\
\hline \multicolumn{3}{|l|}{ Insurance status } \\
\hline Insured & $0.991^{c}$ & $0.987-0.996$ \\
\hline Uninsured & Referent & - \\
\hline \multicolumn{3}{|l|}{ State-level pharmacist privilege } \\
\hline All CDC recommended ${ }^{f}$ & $1.044^{c}$ & $1.040-1.049$ \\
\hline Selected CDC recommended & Referent & - \\
\hline \multicolumn{3}{|l|}{ Vaccination type ${ }^{g}$} \\
\hline CDC recommended & Referent & - \\
\hline Travel/other & $1.166^{c}$ & 1.137-1.195 \\
\hline \multicolumn{3}{|l|}{ Number of vaccines during year } \\
\hline 1 & Referent & - \\
\hline$\geq 2$ & $1.353^{c}$ & $1.343-1.363$ \\
\hline \multicolumn{3}{|l|}{ Pharmacy use } \\
\hline Vaccination only & Referent & - \\
\hline Vaccination and prescription(s) & 1.000 & $0.994-1.005$ \\
\hline
\end{tabular}

$C D C=$ Centers for Disease Control and Prevention.

a From logistic regression analysis; adjusted for all other covariates.

b Calculated from patient's age on date of vaccination.

' Statistically significant.

d Determined from patient zip code and Rural Urban Community Area Codes (RUCA). ${ }^{18}$

e Determined from Medical Condition Codes ascertained from patient prescriptions for chronic medications.

${ }^{f}$ Authorized per protocol or with patient-specific prescription for CDC-recommended vaccines.

9 Vaccinations offered included influenza; herpes zoster (shingles); pneumonia; tetanus, diphtheria, and acellular pertussis (Tdap); typhoid; hepatitis A and/or B (hepatitis); human papillomavirus (HPV); meningitis; measles, mumps, and rubella (MMR); varicella zoster (chicken pox); yellow fever; rabies; polio; and Japanese encephalitis.

Source: Walgreens pharmacy claims data, August 2011 through July 2012.

respectively, before departure to a developing country. ${ }^{26}$ Although these low immunization rates stem in large part from lack of awareness of risk by travelers, they may also be related to primary care clinician recommendations. When compared with primary care clinicians, pharmacists may provide more consistent and accurate recommendations for travel vaccines. ${ }^{27}$
The results of our multivariate model show that patients accessing vaccinations during off-clinic hours were more likely to be aged younger than 65 years. Patients aged younger than 18 years used off-clinic hours for vaccination more than any other age-group in the study (Table 2). According to the CDC, influenza vaccination rates are low for people aged 13 to 17 years (34.5\%) and 18 to 49 years $(29.9 \%)$ compared with those aged 65 years and older (69.6\%). ${ }^{5}$ With the low rates of adult and adolescent vaccination in the United States, community pharmacies are creating new opportunities for vaccination that may help the country achieve the Healthy People 2020 vaccination goals. ${ }^{28} \mathrm{~A}$ study conducted in rural West Virginia suggested that convenience was the primary determining factor in mothers' decisions to take their children to pharmacies for vaccinations. ${ }^{29}$ Expanded hours and access to vaccination for patients aged younger than 18 years may ultimately prevent students from missing school because of illness or because of the need to visit a physician's office during school hours.

Results show that pharmacists are also vaccinating patients aged 65 years and older, who are at higher risk for health complications from vaccine-preventable diseases such as influenza, pneumonia, and herpes zoster. In our sample, approximately $46 \%$ of patients fell into this age-group, with a higher proportion receiving vaccination during traditional clinic hours. This finding may also just represent a population more likely to be retired who already normally access medical care during the day. Using only prescription profiles, pharmacists previously demonstrated their ability to target high-risk populations for pneumococcal vaccine. ${ }^{30}$ Interestingly, for the $40 \%$ of our patients with at least 1 inferred chronic condition, the frequency did not differ greatly whether they were vaccinated during traditional or off-clinic hours, suggesting patients with or without chronic diseases use nontraditional hours for vaccination in a similar manner. A previous study indicated that pharmacists were successful at identifying at-risk patients and providing additional immunization services. ${ }^{30}$ Of patients receiving influenza vaccinations at a community pharmacy, $4.9 \%$ who were also at risk for invasive pneumococcal disease received the vaccination per pharmacist recommendations during the study period. In this study, this rate was higher than a benchmark uptake rate of $2.9 \%$, a rate typically seen at physicians' offices. ${ }^{30}$

In our sample, $94 \%$ of all vaccinations were provided in a metropolitan or micropolitan setting, which most likely reflects the greater availability of pharmacies and those open during off-clinic hours in urban areas. Not all people in urban areas have equal access to health care services, which is an element of the 
Health Resources and Services Administration definition of a Medically Underserved Area (MUA). A previous study found that $43 \%$ of the US population resides within an MUA, and Walgreens pharmacies served nearly one-half of this population; nationwide, nearly one-third of influenza vaccinations were administered by pharmacies located in MUAs. ${ }^{13}$

Unfortunately, pharmacists' ability to fully enhance vaccination rates is limited by a patchwork of state laws that allow either unrestricted authority to administer vaccines or a variety of restrictions. For example, 41 states/territories allow pharmacists to give any vaccine, but 17 restrict pharmacist administration to patients aged 18 years and older. All 50 states, the District of Columbia, and Puerto Rico now at least allow pharmacists to administer influenza vaccinations to all adults aged 18 years or older, however. A 2004 study showed that as more states allowed pharmacists to vaccinate, the immunization rates for those states were higher than the rates in states that did not allow this practice, suggesting pharmacists were not just shifting patient populations from medical clinics, but identifying new populations. ${ }^{31}$ In our study, patients residing in states that authorized pharmacists to administer all CDC-recommended vaccinations were more likely to use off-clinic hours. If states expand pharmacist immunization privileges, patient access and coverage may increase. A previous study estimated that if states were granted full immunization privileges, there would be a $148 \%$ increase in pneumococcal vaccination and a $77 \%$ increase in herpes zoster vaccination. ${ }^{32}$

In our study, there was no significant difference in the use of off-clinic hours for patients only receiving a vaccination vs patients also picking up a prescription. Overall, a relatively small proportion of patients (13.1\%) picked up a prescription medication at the vaccination visit. This pattern may be due to a relatively better overall health status of patients receiving vaccinations and thus a lack of a need to fill any prescriptions, or due to patients seeking immunization services from a pharmacy independent of prescription services. The CDC reports that persons whose last physician's visit for a routine checkup was at least 1 year ago were more likely to receive vaccinations in a nonmedical setting than those whose last physician's visit for a routine checkup was more recent $(53.5 \%$ vs $38.8 \%)$, suggesting that the availability of influenza vaccination in nonmedical settings can complement health care professional efforts by reaching populations less likely to be seen by clinicians. ${ }^{8}$

Few studies have demonstrated this type of offclinic hours access made possible by community pharmacies. One study analyzing retail clinics found that from 2007 to $2009,44.4 \%$ of all visits (including nonimmunization visits) were on the weekend or during weekday hours when physician offices are typically closed..$^{33}$ Previous studies have also indicated that patients trust the pharmacist to administer immunizations and value the ease of access. ${ }^{34}$ Our study builds on the model of pharmacy-based immunization access by exploring patient use of the times pharmacists are available to vaccinate, patient demographics, and pharmacy use.

\section{Limitations}

This study involved only a single pharmacy chain; however, a survey of multiple chain pharmacies indicated that many have also expanded hours and vaccine offerings. ${ }^{35}$ As this was a retrospective pharmacy immunization record review, we could only speculate why patients chose to be vaccinated when they did. According to the previous studies, convenience is likely the greatest driver of use of pharmacy-based immunizations. ${ }^{7,29}$ Second, we used a time period to serve as a proxy for traditional clinic hours, but we did not have data on clinics that might have had expanded hours. Prescription data were used exclusively to infer the presence of a chronic condition in vaccinees, but patients may have received their medications for chronic conditions from a different pharmacy, potentially underestimating the rate of comorbid illnesses; however, when patients seek vaccination, pharmacists use a standardized screening tool that can determine presence of medical conditions. The data we used did not include patient race, which has been demonstrated to be a driver of vaccination uptake. ${ }^{8}$

\section{Implications}

The typical individual who accesses immunization services during off-clinic hours is male, younger, and healthier, and resides in an urban area. When immunization access is expanded, patients will seek and receive vaccinations at a time that is most convenient to them, often when medical clinics are closed. Although those older than 65 years of age continue to represent the largest segment receiving vaccinations in the pharmacy, younger working-aged patients may be more likely to receive vaccinations during off-clinic hours. Expanding the ability of pharmacists to administer all vaccines to adolescents and adults in all states without a physician prescription, coupled with expanded hours of operation, is likely to have the greatest impact on pharmacybased immunization rates.

To read or post commentaries in response to this article, see it online at http://www.annfammed.org/content/11/5/429.

Key words: Immunizations; pharmacy; infectious diseases; vaccines; after-hours care 
Submitted September 7, 2012; submitted, revised, December 28, 2012; accepted January 31, 2013.

Acknowledgments: The authors acknowledge Zhongwen Huang, MS, for his data management support and the more than 26,000 pharmacists who provided vaccinations to the study population.

Funding support: Financial support for this study was provided entirely by Walgreen Co.

\section{References}

1. National Foundation for Infectious Diseases. Facts about adult immunization. August 2009. http://www.nfid.org/publications/factsheets/adultfact.pdf. Accessed May 29, 2012.

2. Centers for Disease Control and Prevention. National, state, and local area vaccination coverage among children aged 19-35 months-United States, 2009. MMWR Morb Mortal Wkly Rep. 2010; 59(36):1171-1177.

3. Centers for Disease Control and Prevention. Adult vaccination coverage-United States, 2010. MMWR Morb Mortal Wkly Rep. 2012; 61(4):66-72.

4. Centers for Disease Control and Prevention. Estimates of deaths associated with seasonal influenza-United States, 1976-2007. MMWR Morb Mortal Wkly Rep. 2010;59(33):1057-1062.

5. Centers for Disease Control and Prevention. Final state-level influenza vaccination coverage estimates for the 2010-11 season-United States, National Immunization Survey and Behavioral Risk Factor Surveillance System, August 2010 through May 2011. 2011. http://www.cdc.gov/flu/professionals/vaccination/ coverage_1011estimates.htm. Accessed May 28, 2012.

6. Freed GL, Clark SJ, Cowan AE, Coleman MS. Primary care physician perspectives on providing adult vaccines. Vaccine. 2011;29(9): 1850-1854.

7. Postema AS, Breiman RF; National Vaccine Advisory Committee. Adult immunization programs in nontraditional settings: quality standards and guidance for program evaluation. MMWR Recomm Rep. 2000;49(RR-1):1-13.

8. Centers for Disease Control and Prevention. Place of influenza vaccination among adults-United States, 2010-11 influenza season. MMWR Morb Mortal Wkly Rep. 2011;60(23):781-785.

9. Crawford ND, Blaney S, Amesty $S$, et al. Individual- and neighborhood-level characteristics associated with support of in-pharmacy vaccination among ESAP-registered pharmacies: pharmacists' role in reducing racial/ethnic disparities in influenza vaccinations in New York City. J Urban Health. 2011;88(1):176-185.

10. Hogue MD, Grabenstein JD, Foster SL, Rothholz MC. Pharmacist involvement with immunizations: a decade of professional advancement. J Am Pharm Assoc (2003). 2006;46(2):168-179; quiz 179-182.

11. Uscher-Pines L, Maurer J, Kellerman A, Harris KM. Healthy young and middle age adults: what will it take to vaccinate them for influenza? Vaccine. 2010;28(46):7420-7422.

12. Westrick SC. Pharmacy characteristics, vaccination service characteristics, and service expansion: an analysis of sustainers and new adopters. J Am Pharm Assoc (2003). 2010;50(1):52-61.

13. Murphy PA, Frazee SG, Cantlin JP, Cohen E, Rosan JR, Harshburger $D E$. Pharmacy provision of influenza vaccinations in medically underserved communities. J Am Pharm Assoc (2003). 2012;52(1):67-70.

14. Francis M, Hinchliffe A. Vaccination Services Through Community Pharmacy: A Literature Review. Cardiff, Wales, United Kingdom: Public Health Wales; 2010.

15. Schoen C, Osborn R, Huynh PT, Doty M, Peugh J, Zapert K. On the front lines of care: primary care doctors' office systems, experiences, and views in seven countries. Health Aff (Millwood). 2006;25(6):w555-w571.
16. US Office of Personal Management. Operating Status \& Schedules. 2011. http://www.opm.gov/operating_status_schedules/fedhol/2011. asp. Accessed Nov 21, 2012.

17. Medi-Span. Drug Indications Database: Documentation Manual. Indianapolis, IN: Wolters Kluwer Health, Inc; 2003.

18. Morrill RCJ, Hart LG. Metropolitan, urban, and rural commuting areas: toward a better depiction of the U.S. settlement system. Urban Geogr. 1999;20(8):727-748.

19. Skelton JB. Pharmacist-provided immunization compensation and recognition: white paper summarizing APhA/AMCP stakeholder meeting. J Am Pharm Assoc (2003). Nov-Dec 2011;51(6):704-712.

20. Advisory Committee on Immunization Practices. Recommended immunization schedules for persons aged 0 through 18 yearsUnited States, 2012. MMWR Morb Mortal Wkly Rep. 2012;61(5):1-4. Corrected in MMWR Morb Mortal W/kly Rep. 2012 2;61(8):147. (Note: Dosage error in article text).

21. Advisory Committee on Immunization Practices. Recommended adult immunization schedule: United States, 2012. MMWR Morb Mortal W/kly Rep. 2012;61(4)1-7.

22. United States Department of Labor Bureau of Labor Statistics. Workers on Flexible and Shift Schedules in 2004, Summary. 2005. http://www.bls.gov/news.release/flex.nr0.htm. Accessed Nov 15, 2012.

23. Nichol KL, Mallon KP, Mendelman PM. Cost benefit of influenza vaccination in healthy, working adults: an economic analysis based on the results of a clinical trial of trivalent live attenuated influenza virus vaccine. Vaccine. 2003;21(17-18):2207-2217.

24. Eames KTD, Brooks-Pollock E, Paolotti D, Perosa M, Gioannini C, Edmunds WJ. Rapid assessment of influenza vaccine effectiveness: analysis of an Internet-based cohort. Epidemiol Infect. 2012;140 (7):1309-1315.

25. Lynch MF, Blanton EM, Bulens S, et al. Typhoid fever in the United States, 1999-2006. JAMA. 2009;302(8):859-865.

26. Hamer DH, Connor BA. Travel health knowledge, attitudes and practices among United States travelers. J Travel Med. 2004;11(1):23-26.

27. Durham MJ, Goad JA, Neinstein LS, Lou M. A comparison of pharmacist travel-health specialists' versus primary care providers' recommendations for travel-related medications, vaccinations, and patient compliance in a college health setting. J Travel Med. 2011;18(1):20-25.

28. Healthy People 2020. US Department of Health and Human Services. Office of Disease Prevention and Health Promotion. http:// www.healthypeople.gov/2020/default.aspx. Accessed May 30, 2012.

29. Ndiaye SM, Madhavan S, Washington ML, et al. The use of pharmacy immunization services in rural communities. Public Health. 2003;117(2):88-97.

30. Taitel M, Cohen E, Duncan I, Pegus C. Pharmacists as providers: targeting pneumococcal vaccinations to high risk populations. Vaccine. 2011;29(45):8073-8076.

31. Steyer TE, Ragucci KR, Pearson W/S, Mainous AG III. The role of pharmacists in the delivery of influenza vaccinations. Vaccine. 2004; 22(8):1001-1006.

32. Taitel MS, Fensterheim LE, Cohen ES, Cannon AE. Improving pneumococcal and herpes zoster vaccination uptake: expanding pharmacist privileges. Am J Manag Care. 2013;19(9):e309-e313.

33. Mehrotra A, Lave JR. Visits to retail clinics grew fourfold from 2007 to 2009, although their share of overall outpatient visits remains low. Health Aff (Millwood). 2012;31(9):2123-2129.

34. Grabenstein JD, Guess HA, Hartzema AG. People vaccinated by pharmacists: descriptive epidemiology. J Am Pharm Assoc (2003). 2001;41(1):46-52.

35. Pilisuk T, Goad J, Backer H. Vaccination delivery by chain pharmacies in California: results of a 2007 survey. J Am Pharm Assoc (2003). 2010;50(2):134-139. 